\title{
9. Brain bioelectrical function and cerebral oxygenation during neonatal anaesthesia with two different opioid drugs - pilot clinical study.
}

\author{
Karpinski, Lukasz T.'; Gaca, Michal2; Miechowicz, Izabela³; Moczko, Jerzy3; Mielnik, Katarzyna'; Matusiak, Grzegorz; \\ Jerzak, Agata'; Mazela, Jan'1. \\ 1. Dept of Neonatology and Infectious Diseases, Poznan University of Medical Sciences, Poznan, Poland. 2. Dept of \\ Anesthesiology in Obstetrics and Gynecology, Poznan University of Medical Sciences, Poznan, Poland. 3. Dept of \\ Computer Sciences and Statistics, Poznan University of Medical Sciences, Poznan, Poland.
}

\section{BACKGROUND AND AIMS}

Preterm newborns are often in need of surgical treatment, which is strictly connected with administration of anesthetics. One of the most commonly used drugs in newborn anesthesia are opioids. Their pharmacological action might cause hypotension, respiratory failure and depression of the central nervous system [1,2].

Still there is a limited number of studies evaluating influence of an anesthesia on a preterm newborn. One of the most commonly used opioids are fentanyl and remifentanyl. They have different pharmacokinetics (table 1.) $[3,4]$ and they might have different effect on brain function.

There are two new methods of brain function monitoring. Amplitude integrated electroencephalography (aEEG) which allows to continuously monitor electric brain function. Near Infrared Spectroscopy (NIRS) monitors brain tissue oxygenation.

The aim of our study is to use these two methods combined with classical pulsoxymetry to monitor preterm newborns during anaesthesia.

\section{METHODS}

Preterm patients born between 2015 and 2016 requiring photocoagulation or ranivizumab injection due to retinopathy of prematurity were included into the study.

Patients were assigned to one of the two groups. Group one received remifentanil during the anaesthesia and group two received fentanyl. Each patient was mechanically ventilated during the procedure and was extubated after it, when patients own breathing was present and spontaneous movement reappeared.

Each patient was monitored with aEEG, NIRS and pulseoximetry. For aEEG we used CCFM Digitrack (Elmiko, Poland). From aEEG following parameters were studied: mean aEEG voltage and interburst intervals (IBI). For brain oxygenation (NIRS) we used INVOS 5100C (Covidien, USA). For pulsoximetry we used NONIN, which is integrated with aEEG transducer (USA). Lenght of ventilatory suport after the procedurę was also analyzed. Each patient was monitored for 5 minutes before the surgery, during the surgery and for 1 hour and 30 minutes after the procedure. During surgery 25 minutes of stable signal was chosen. Time after procedure was divided into 3 epoch (30minutes each) for the analysis.

\begin{tabular}{|r|r|r|}
\hline Onset & Fentanyl & Remifentyl \\
Duration & $0,5-1$ hour & $1-3$ minutes \\
\hline Metabolism & $\begin{array}{r}\text { Rapid via blood and } \\
\text { tissue esterases }\end{array}$ & $\begin{array}{r}\text { Hepatic, primarily via CYP3A4 by N- } \\
\text { dealkylation (to norfentanyl) and } \\
\text { hydroxylation to other inactive } \\
\text { metabolites }\end{array}$ \\
& 2,4 hours & 5,4 minutes
\end{tabular}

Half elimination

gs used in the study

\section{RESULTS}

15 patients had been included into group 1 and 13 into group 2 .

Patients in group 2 had longer time on mechanical ventilation after the procedure (505min. vs. $172 \mathrm{~min} . \mathrm{p}=0,004)$.
For saturation and brain oxygenation there were no differences between groups before, during and after the procedure (Figure 1).

Patients in the group 1 had significantly higher heart rate in first 30 minutes after the surgery $(p=0,004)$ and in last 30 minutes $(p=0,03)$ (Figure 1.).

Patients in group 2 had lower mean voltage in first 30 minutes after the procedure $(p=0,008)$ and during last 30 evaluated minutes $(p=0,04)$ (Figure 1).

Considering IBIs there were no differences between groups before $(p=0,155)$ and during the procedure $(p=0,055)$.

After the surgery, in all three phases there were significant differences between the groups. Patient that received fentanyl had more IBIs after the surgery.

\section{CONCLUSIONS}

Infants receiving remifentanil needed 3 times less time on mechanical ventilation after the surgery when compared to fentanyl. They had also higher aEEG voltage and more continuous signal after the procedure. Difference in heart rate requires further investigation.
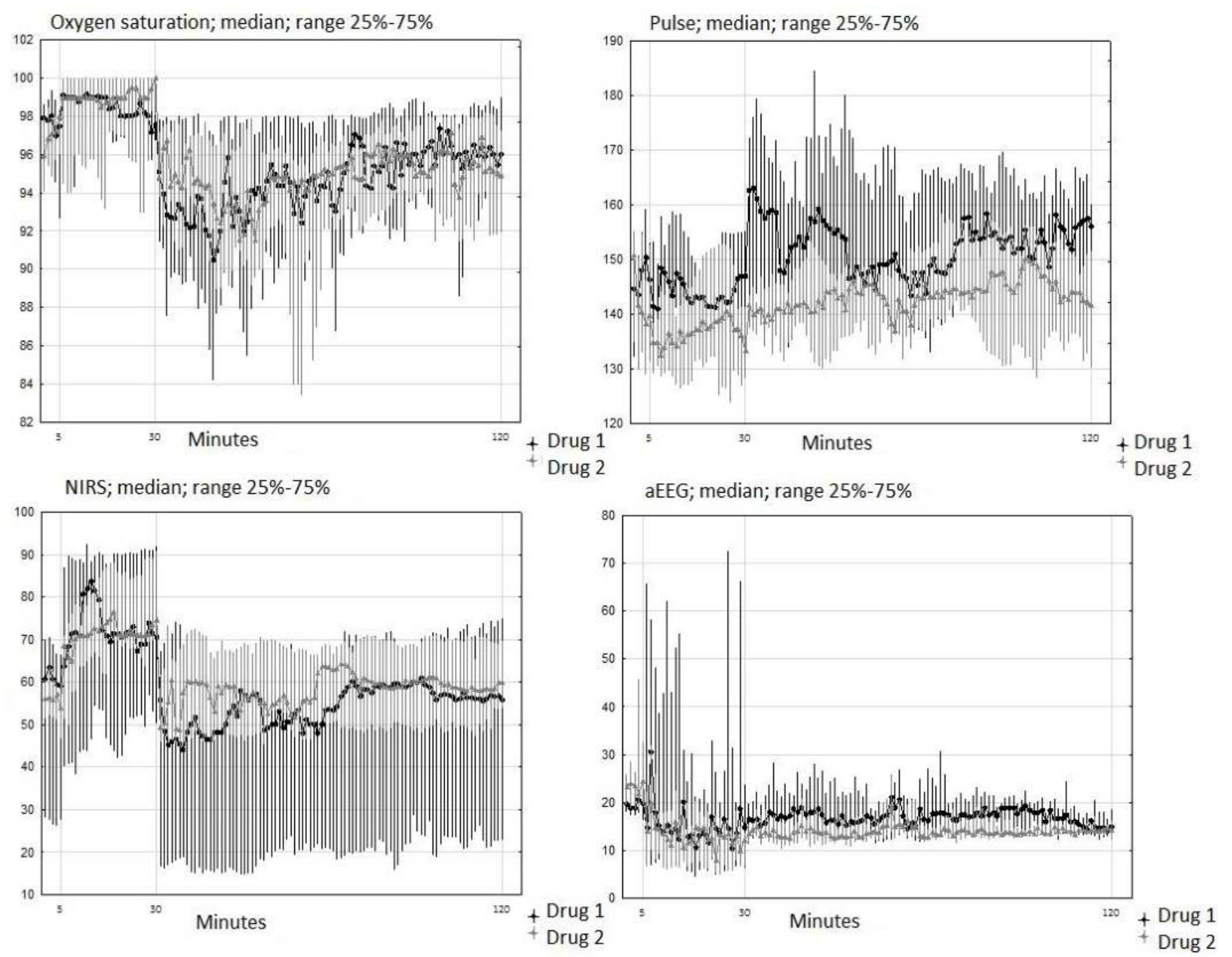

Figure 1. Comparison of saturation, pulse, brain oxygenation and mean aEEG voltage between groups.

\section{REFERENCES}

1.Bell AH, Greisen $G$, Pryds O. Comparison of the effects of phenobarbitone and morphine administration on EEG activity in preterm babies. Acta Paediatr 1993;82:35-9.

2. Nguyen The Tich S, Vecchierini MF, Debillon T, Péréon Y. Effects of sufentanil on electroencephalogram in very and extremely preterm neonates. Pediatrics 2003;111:123-8.

3.https://www.uptodate.com/contents/fentanyl-pediatric-druginformation/print?source=search_result\&search=fentanyl\&selectedTitle $=2 \sim 150$

4.www.uptodate.com/contents/remifentanil-pediatric-druginformation?source=search_result\&search=remifentanil\&selectedTitle= 2 74 